\title{
Multiband Multistatic Synthetic Aperture Radar for Measuring Ice Sheet Basal Conditions
}

\author{
John Paden, Shadab Mozaffar, David Dunson, Chris Allen, Sivaprasad Gogineni, Torry Akins \\ Radar Systems and Remote Sensing Laboratory \\ University of Kansas \\ Lawrence, KS, USA \\ paden@ittc.ku.edu
}

\begin{abstract}
Ice sheet models are necessary to understand ice sheet dynamics and to predict their behavior. Of the primary inputs to these models, basal conditions are the least understood. By observing the forward and backscatter across a wide frequency range (over two octaves) the basal conditions can be established with a high level of confidence. For this purpose, we developed a multistatic synthetic aperture radar system that operates on three frequency bands (75-85 MHz, 140-160 MHz, and 330-370 MHz). The radar system is designed to use pulse compression techniques and coherent integration to obtain high loop sensitivity $(203 \mathrm{~dB})$ necessary to overcome radio frequency losses in ice. The system will be tested at Summit, Greenland $\left(7^{\circ} 34^{\prime}\right.$ N, 38²9' W) during July 2004.
\end{abstract}

Keywords- ice, scattering, multistatic, synthetic aperture radar

\section{INTRODUCTION}

International interest in Earth's climate future is growing, as an increasing number of data sources and models point toward the possibility of global climate change [1]. Understanding and predicting global climate change requires knowledge of the cyrosphere and its interaction with climate. One potentially devastating effect of climate change is an increase in sea level. Coastal region resources and habitats are very sensitive to sea level rise and also tend to be population centers [2]. To understand how the cyrosphere interacts with the climate, models of ice flow are required.

To model ice flow, the boundary conditions of the ice must be known with a high level of accuracy. A fundamental boundary condition in ice flow modeling is the basal condition of the ice [3]. The two basal properties of most interest are (1) the existence of liquid water and (2) the basal surface roughness. Since electromagnetic scattering and reflection are sensitive to these properties, radar remote sensing is a potential solution. Additionally, ice flow models require wide area coverage rendering brute force techniques, such as drilling boreholes, inadequate.

We have developed a multi-frequency multistatic synthetic aperture radar for measuring polar ice sheet basal conditions. The multi-frequency operation is intended to help isolate the bedrock reflection from other propagation effects and to distinguish various basal properties.

This work was supported in part by the National Science Foundation grant OPP-0122520 and by the National Aeronautics and Space Administration grant NGT5-30449.
The radar system has a single transmitter and two receivers. One receiver (monostatic receiver) is collocated with the transmitter and operates as a side-looking synthetic aperture radar which measures backscatter from the ice sheet base. The second receiver (bistatic receiver) is positioned so that it receives the forward scatter from the same location of the ice sheet base. The combination of these two techniques allows for more efficient coverage of the ice sheet [4]. Furthermore, simultaneous measurements of backscatter and forward scatter provide additional insight into the basal conditions.

The radar system uses an arbitrary waveform generator for pulse generation. The transmission pulse used currently is a modified linear FM chirp. The chirp was modified to compensate for transmitter imperfections (specifically non-flat gain which affects signal-to-noise ratio). The received signal is recorded uncompressed using offset video, which preserves the coherent nature of the signal.

The radar system is described in section II, including a description of the bistatic synchronization technique. The link budget and radar performance analysis is described in section III. Finally, laboratory results are shown in section IV.

\section{SYSTEM DESCRIPTION}

\section{A. Hardware Description}

Fig. 1 illustrates the system flow of control, timing, and data signals. The bistatic system has been excluded, but is the same as the system shown without the arbitrary waveform generator (AWG) and transmitter. The pulse repetition frequency $(\mathrm{PRF})$ generator sets the timing for the entire system and is referenced off a $10 \mathrm{MHz}$ Rubidium source. The pulse

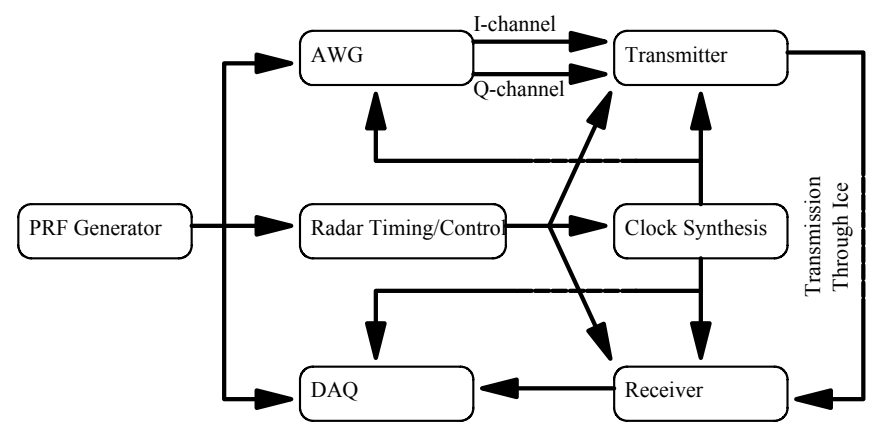

Figure 1. System flow diagram. 
trigger, data capture trigger, radar timing/control signals, etc. are all specified in reference to the PRF signal. The radar timing and control signals are all digital. This allows the state of the radar to easily be recorded with the data.

The AWG and DAQ both run at $120 \mathrm{MHz}$ and receive their clocks from the clock synthesis board. The arbitrary waveform generator (AWG) utilizes a two-channel 16 bit digital-toanalog converter running at $120 \mathrm{MHz}$. The AWG can store waveforms with up to 262,144 samples $(2.2 \mathrm{~ms}$ at $120 \mathrm{MHz}$ ). The spurious free dynamic range of the AWG is $73 \mathrm{~dB}$. The data acquisition system (DAQ) records data with 12 bits of accuracy with an effective noise floor of $-62 \mathrm{dBm}$. The DAQ can perform up to 1 million coherent integrations before passing data back to the computer system. The AWG and DAQ can also perform zero-pi interpulse modulation to reduce coherent noise in the system.

In addition to the AWG and DAQ clocks, the clock synthesis board generates the local oscillator (LO) signal for both the transmitter and the receiver using a direct digital synthesizer (DDS). The DDS is run off a $1 \mathrm{GHz}$ clock that is generated from the $10 \mathrm{MHz}$ Rubidium source. The LO frequencies are $120 \mathrm{MHz}$ for the $75-85 \mathrm{MHz}$ band, $120 \mathrm{MHz}$ for the $140-160 \mathrm{MHz}$ band, and $320 \mathrm{MHz}$ for the $330-370 \mathrm{MHz}$ band.

The signal generated by the AWG and the signal passed to the DAQ are offset video signals with a center frequency of 30 $\mathrm{MHz}$. To properly reject unwanted signals, a single side band modulator is used in the transmitter. This requires an I and Q channel input. The relative phase of the I and Q channels from the AWG chooses the side band desired (lower side band for the lowest frequency band and upper side band for the two higher frequency bands).

Fig. 2 shows the block diagram of the transmitter. After modulating the offset video signal up to the carrier frequency, the signal is filtered in the band-pass-filter bank. It is then amplified twice in the preamplifier stage to the level needed for the power amplifier. Since the power amplifier has about $10 \mathrm{~dB}$ of gain variation between the bands, different amounts of attenuation for each band have been added in the band-passfilter bank. After the pre-amplifier stage is a transmit-mod switch, which turns the transmitter off while not transmitting.

The power amplifier amplifies the signal to $100 \mathrm{~W}$. For the July 2004 field test, a log-periodic antenna will be used. The return loss of the antenna is greater than $10 \mathrm{~dB}$ in each frequency band.

Attenuator pads have been added for devices with poor impedance matches. These include the reflective filters used in the filter banks, the mixer, and the transmit amplifier.

Fig. 3 shows the block diagram of the receiver. An identical log-periodic antenna will receive the signal. The low noise amplifier (LNA) is preceded by a built-in limiter (maximum signal power of $30 \mathrm{dBm}$ ) to prevent damage to the LNA. The LNA has a fast recovery time of 1.5 us after saturation. The LNA is followed by a high-isolation receiver-blanking switch, which protects the rest of the receiver from being saturated during transmission. The LNA was placed before the switch to improve the noise figure of the system. The receiver-blanking switch is not necessary in the bistatic receiver due to the separation and associated spherical spreading loss, but is required for the monostatic receiver.

After the blanking switch, there is an attenuator pad and the filter bank. Attenuator padding was added after the receiverblanking switch due to video leakage of the control signal onto the radio frequency (RF) signal and the reflective nature of the band pass filters. The filter bank is followed by a digital step attenuator. The step attenuator can be set from 5-35 dB in $5 \mathrm{~dB}$

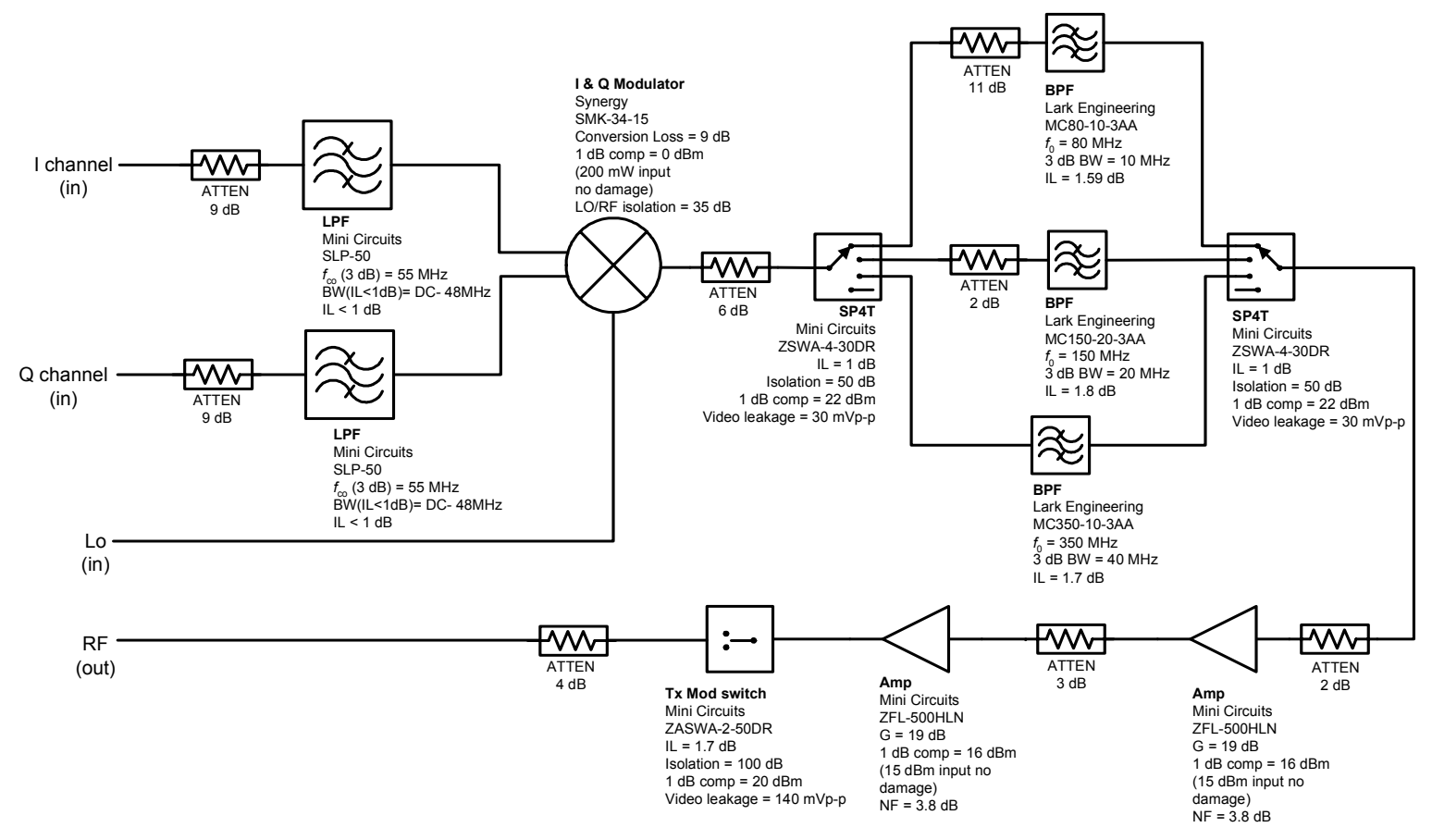

Figure 2. Transmitter block diagram. 


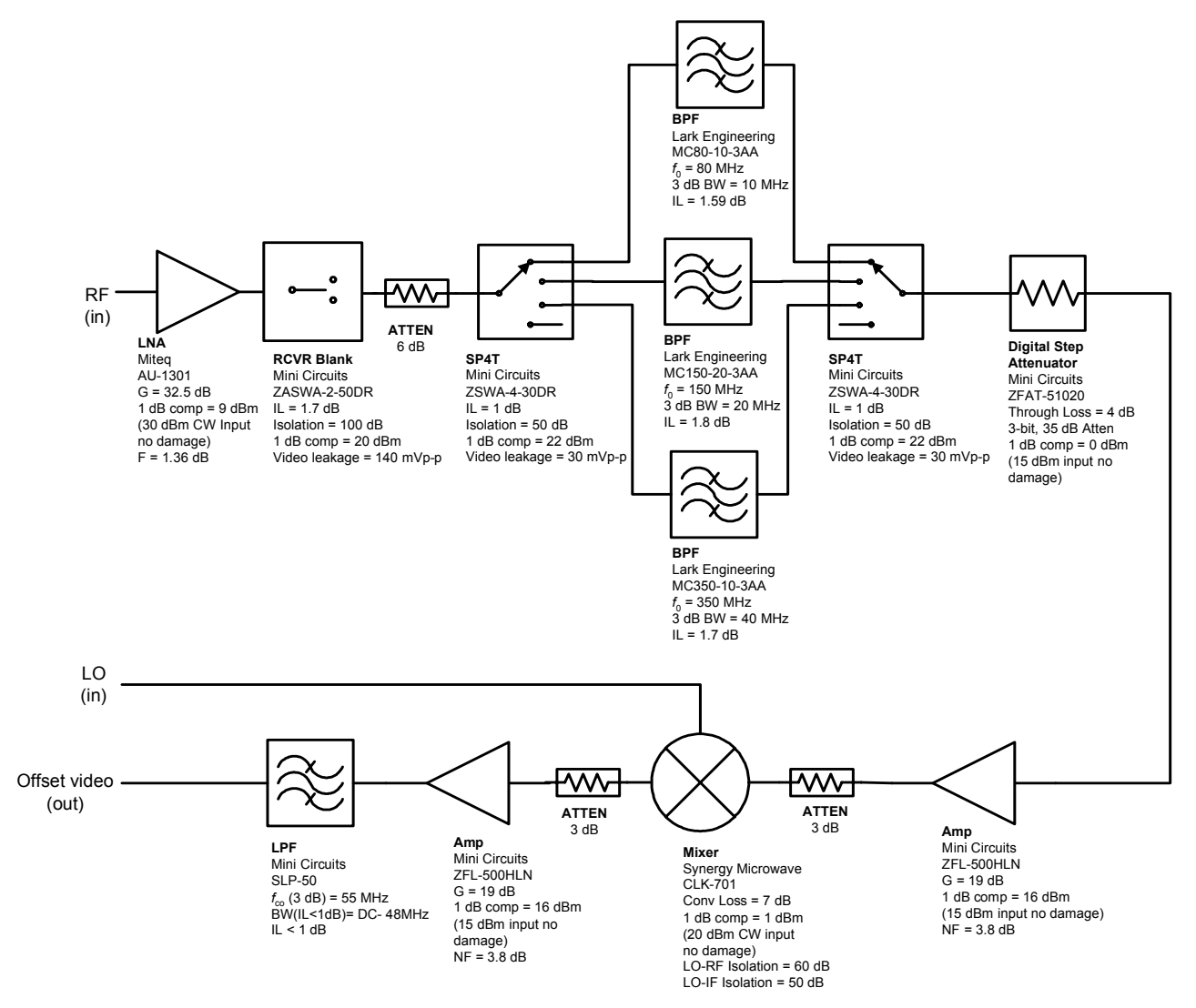

Figure 3. Receiver block diagram.

steps. The signal is then amplified, mixed down to offset video, and then amplified again. The amplified signal is passed through an anti-aliasing low pass filter before being recorded by the DAQ. Attenuator pads were also added around the mixer to compensate for poor impedance matches.

\section{B. Bistatic Receiver Synchronization}

For the bistatic receiver to operate, it must synchronize control and timing signals with the transmitter. It also needs to provide phase synchronization even when the recorded signal of interest is $30-50 \mathrm{~dB}$ beneath the noise floor. This is provided through two mechanisms. The first is an IEEE 802.11 wireless network that provides a link to synchronize the radar control parameters such as pulse repetition frequency, transmit waveform, position and heading information, etc. The second mechanism provides the timing synchronization (locking the PRF signal and locking the phase). To do this, both the monostatic transmitter and bistatic receiver contain a highly stable, programmable, $10 \mathrm{MHz}$ Rubidium source. Additionally, the PRF signal is programmable.

The radar control parameters are transported through the network so that each end of the system (transmitter and bistatic receiver) knows what the transmitted pulse will be, what frequency band is being operated on, and the PRF. After the transmitter begins transmitting, the bistatic receiver software scans the entire pulse period (equal to the time between pulses) into a temporary buffer by adjusting the offset of the data capture trigger in reference to the PRF signal. Once the entire pulse period has been captured, the temporary buffer is analyzed to find the direct path signal. Then, using highprecision position and heading information, the range between transmit and receive antennas is found. This range is then used to offset the location of the direct path signal in the data capture window so that the bistatic receiver's PRF signal is aligned with the transmitter's PRF signal.

Once the PRF signals are locked, the bistatic receiver software begins monitoring the phase and range bin of the direct path signal. As the radar system moves, new range and heading information is used to provide accurate range information to the software. Using this range information and the phase/range-bin of the direct path signal, the Rubidium source's frequency is adjusted until the unexpected phase/range-bin drift in the direct path is zero. After phase locking, the bistatic software allows data to be recorded to disk, but continues monitoring the direct-path signal and making adjustments to the Rubidium source as needed.

The phase synchronization requires a very accurate estimate of the range between the transmit and receive antennas. This requirement is especially stringent in the highest frequency band (330-370 MHz), which has a free space wavelength of 81 $\mathrm{cm}$. To provide this quality of ranging information, a differential global positioning system and gyro are used. Together these provide position information with an error standard deviation of $1 \mathrm{~cm}$ in latitude and longitude, and $2 \mathrm{~cm}$ in elevation, and heading information to within 1 degree.

\section{RADAR PERFORMANCE ANALYSIS}

The loop sensitivity of the radar system in $\mathrm{dB}$ is given by 


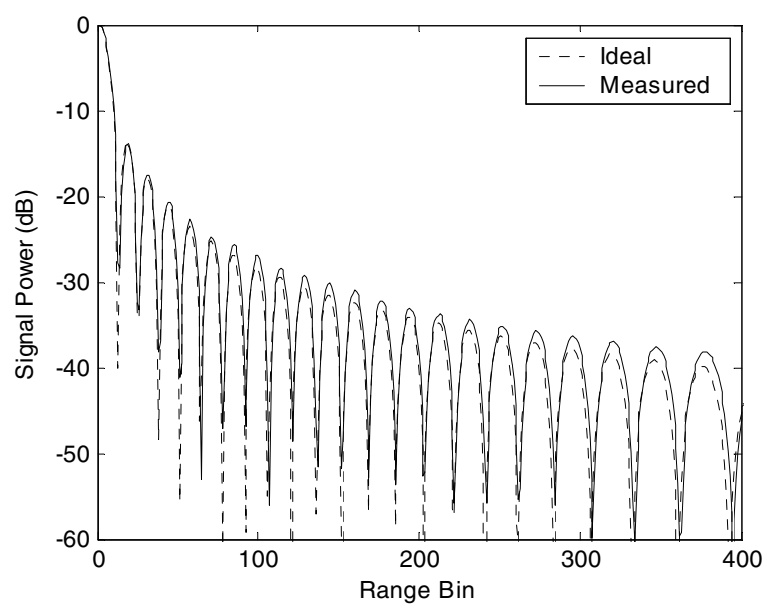

Figure 4. Comparison of ideal and measured matched filtering.

$$
L=P_{T}-10 \log _{10}\left(k T B F_{R C V R}\right)+C+I=203 \mathrm{~dB}
$$

where $P_{T}$ is the transmit power in $\mathrm{dB}, k=1.38 \times 10^{-23}$ is Boltzmann's constant, $T=298 K$ is the reference temperature, $B=40 \mathrm{MHz}$ is the bandwidth of the system, $F_{R C V R}=1.43 \mathrm{~dB}$ is the noise figure of the receiver, $C=10 \log _{10}(10 e-6 \cdot 40 e 6)$ $=26 \mathrm{~dB}$ is the compression gain with a $10 \mu \mathrm{s}$ pulse with 40 $\mathrm{MHz}$ of bandwidth, and $I=30 \mathrm{~dB}$ is the integration gain with 1000 averages. Note that as the bandwidth is decreased the noise input into the receiver goes down as the compression gain goes down so the loop sensitivity does not change.

When the receiver is in the maximum gain state, the largest signal into the receiver without saturating is $-38 \mathrm{dBm}$. This corresponds to a $4 \mathrm{dBm}$ signal out of the receiver. Fullscale for the DAQ is $4 \mathrm{dBm}( \pm 0.5 \mathrm{~V})$. The maximum gain of the receiver was set to raise the thermal noise floor of the receiver $10 \mathrm{~dB}$ above the noise floor of the DAQ.

The noise figure of the receiver is given by

$$
F_{R C V R}=F_{1}+\frac{F_{2}-1}{G_{1}}+\cdots+\frac{F_{N}-1}{G_{1} G_{2} \cdots G_{N-1}}
$$

(a)
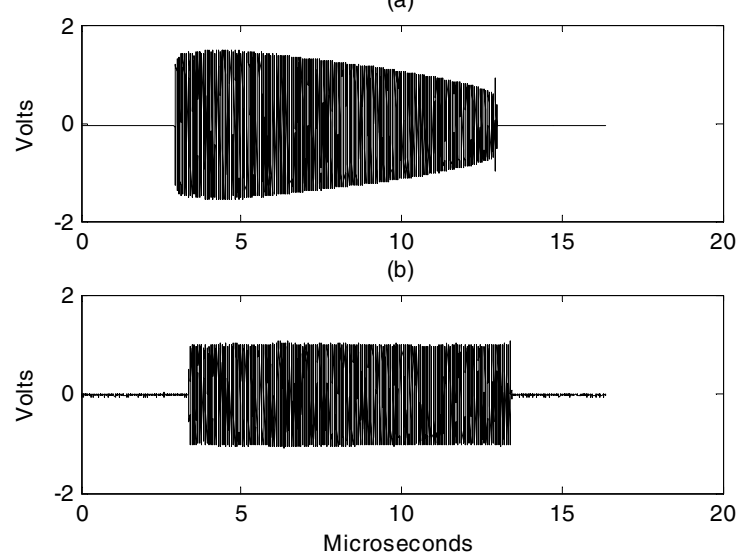

Figure 5. (a) Ideal pulse passed through the transmitter. (b) Predistorted pulse passed through the transmitter.

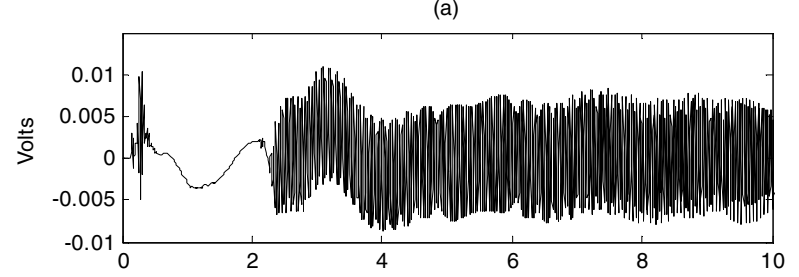

(b)

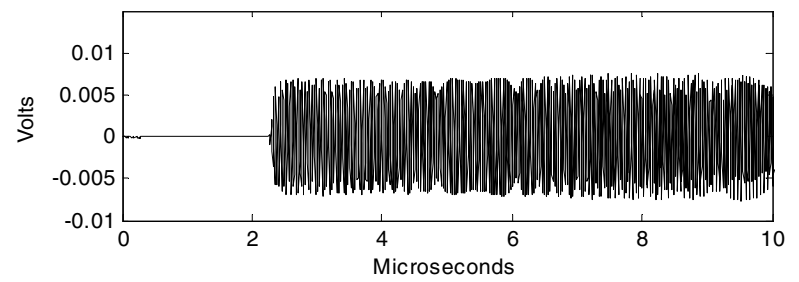

Figure 6. (a) Without zero-pi modulation. (b) With zero-pi modulation.

where $F_{i}$ and $G_{i}$ correspond to the noise figure and gain of the $i^{\text {th }}$ component in the receiver chain. For this receiver, the LNA is the first device with $F_{1}=1.16 \mathrm{~dB}$ and $G_{1}=32.5 \mathrm{~dB}$. The noise figure of the entire system in the maximum gain setting, based on (2), is $1.43 \mathrm{~dB}$.

\section{RESULTS}

The matched filter results from the radar are shown in fig. 4 along with the match filter results from an ideal chirp. There is only a slight discrepancy between the two results. The matched filter coefficients are taken from the complex conjugate of the Fourier transform of a loopback measurement with the system.

The transmit pulse as noted is a linear FM chirp. The transmit amplifier and various filters in the transmitter have non-flat gain. An ideal linear FM chirp is shown in fig 5a. after it has passed through the transmitter. Based on the distortion measured, the transmit waveform was pre-distorted to compensate for the non-flat gain. The result of this waveform after passing through the transmitter is shown in fig $5 \mathrm{~b}$.

The GaAs receiver blanking switch produces a $30 \mathrm{mV}$ positive transient on the RF signal. Since this transient is coherent, we implemented zero-pi modulation which switches the pulse-to-pulse phase of the transmit signal by 180 degrees. The coherent integrator in hardware adds or subtracts the signal based on the phase so that the signal remains coherent. Any coherent noise is then subtracted out since its phase does not change. Figure $6 \mathrm{a}$ and $6 \mathrm{~b}$ show a loopback test without zero-pi modulation and with zero-pi modulation.

\section{REFERENCES}

[1] Kennedy, Donald, "Climate change and climate science," Science, vol. 34, pp. 1565, June 11, 2004.

[2] Douglas, Bruce C., and Richard W. Peltier, "The puzzle of global sealevel rise," Physics Today, pp. 35-40, 2001.

[3] Van der Veen, Cornelis J., Fundamentals of glacier dynamics, A. A. Balkema, Rotterdam, Netherlands, 1999.

[4] Paden, John D., Bistatic/monostatic synthetic aperture radar for ice sheet measurements, M.S. Thesis, University of Kansas, 2003. 